\title{
ARTIGOS
}

Submetido 04.07.2019. Aprovado 18.02.2020

Avaliado pelo sistema double blind review. Editor Científico: Antonio Padula

Versão original

DOI: http://dx.doi.org/10.1590/So034-759020200402

\section{INOVAÇÃO NA CADEIA REVERSA DE RESÍDUOS ELETROELETRÔNICOS: UM ESTUDO SOBRE OS SISTEMAS DE INFORMAÇÃO E AS TECNOLOGIAS DE RASTREAMENTO}

\author{
Innovation in the reverse chain of electrical and electronic waste: $A$ study on \\ information systems and tracking technologies \\ Innovación en la cadena inversa de residuos electroelectrónicos: Un estudio \\ sobre sistemas de información y tecnologías de seguimiento
}

\section{RESUMO}

Esse trabalho tem como objetivo analisar a contribuição de sistemas de informação e as tecnologias de rastreamento para a logística reversa de cadeias de resíduos de eletroeletrônicos. A pesquisa foi realizada por meio de um estudo de caso incorporado em uma cadeia de eletroeletrônicos no contexto brasileiro. Os resultados da pesquisa mostraram que as tecnologias e sistemas implementados na logística direta não são utilizados na logística reversa. Mesmo reconhecendo as tecnologias de rastreamento como uma alternativa para tornar mais eficientes a desmontagem e a reciclagem, prevalece uma visão de aumento de custos, que não contribui para o aumento de vendas e a lucratividade. Infere-se que a resistência dos representantes do setor de eletroeletrônicos em aderir ao acordo setorial, assinado apenas no final de 2019 e estabelecido pela Política Nacional de Resíduos Sólidos (PNRS) em 2010, contribuiu para que empresas adiassem investimentos em sistemas de informação e tecnologias de rastreamento no Brasil.

PALAVRAS-CHAVE | Inovação ambiental, tecnologias de rastreamento, cadeia de suprimentos, logística reversa, resíduos eletroeletrônicos.

\section{ABSTRACT}

This study analyzes the contribution of information systems and tracking technologies to the reverse logistics of electronic and electrical waste chains. The research was a case study in the Brazilian context, incorporating the electronics supply chain. The results show that reverse logistics do not use the technologies and systems implemented in direct logistics. Although tracking technologies can make disassembly and recycling more efficient, the possibility of increased costs remains, and does not contribute to increased sales and profitability. Furthermore, resistance by the representatives of the electronics sector to adhere to the sectoral agreementestablished by the National Solid Waste Policy (PNRS) in 2010 but signed only at the end of 2019-contributed to companies postponing investments in information systems and technologies tracking in Brazil.

ODAir KEYWORDS / Environmental innovation, tracking technologies, supply chain, reverse logistics, waste electrical and electronic.

\section{MARIA TEREZA SARAIVA DE SOUZA ${ }^{1}$}

mtereza@fei.edu.br

0000-0003-4514-2021

\section{JACQUES DEMAJOROVIC1}

jaquesd@fei.edu.br

0000-0001-6131-8790

${ }^{1}$ Centro Universitário da FEI, Departamento de Administração, São Paulo, SP, Brasil

\section{RESUMEN}

El objetivo de este trabajo es analizar la contribución de los sistemas de información y las tecnologías de seguimiento a la logística inversa de las cadenas de desechos electrónicos. La investigación se realizó a través de un estudio de caso incorporado en una cadena de electrónica en el contexto brasileño. Los resultados de la investigación mostraron que las tecnologías y sistemas implementados en logística directa no se utilizan en logística inversa. Incluso reconociendo las tecnologías de seguimiento como una alternativa para hacer que el desensamble y el reciclaje sean más eficientes, prevalece una visión del aumento de los costos, que no contribuye a aumentar las ventas y la rentabilidad. Se infiere que la resistencia de los representantes del sector de electrónica a adherirse al acuerdo sectorial, firmado solo a fines de 2019 y establecido por la Política Nacional de Residuos Sólidos (PNRS) en 2010, contribuyó a que las empresas pospusieran inversiones en sistemas de información y tecnologías de seguimiento en Brasil.

PALABRAS CLAVE / Innovación ambiental, tecnologías de rastreo, cadena de suministro, logística inversa, residuos eléctricos y electrónicos. 


\section{INTRODUÇÃO}

Os processos de obsolescência têm estimulado os consumidores a trocarem com mais frequência os equipamentos eletrônicos como smartphones, tablets, computadores pessoais e jogos eletrônicos, contribuindo para o aumento do descarte desses produtos. Por conterem substâncias com elevado potencial de gerar danos à saúde e ao meio ambiente, em caso de descarte inadequado, as leis ou diretrizes têm forçado os fabricantes a buscarem soluções no desenvolvimento de programas de logística reversa (LR) mais eficientes (Demajorovic, Augusto, \& Souza, 2016)

A melhoria de processo para implantação da $L R$ e da Green Supply Chain Management (GSCM) está relacionada aos investimentos em sistemas de informação (Ajamieh, Benitez, Braojos, Gelhard 2016) para o gerenciamento e compartilhamento das informações ambientais na cadeia (Olorunniwo \& Li, 2010). A tecnologia da informação (TI) emergiu como uma das melhores ferramentas para a inovação tecnológica implementada na cadeia de suprimentos, constituindo-se em um tema de pesquisa emergente na literatura de gestão da cadeia (Hazen \& Byrd, 2012).

A dificuldade principal está no fluxo reverso, no intercâmbio de informação entre os fabricantes e os locais de desmontagem. $\mathrm{O}$ uso de tecnologia de rastreamento, como identificação por radiofrequência (Kumar, 2014), código de barras (Musa, Gunasekaran, \& Yusuf, 2014) ou chips integrados (Um, Stroud, \& Suh, 2015), contribui na triagem e classificação para a reutilização, a desmontagem, a remanufatura ou a reciclagem de equipamentos (Nowakowski, 2018), melhorando a eficiência do processo e reduzindo custos.

Mesmo considerando as vantagens do uso da Tl em programas de LR, esse tema é ainda pouco explorado pela literatura acadêmica (Toyasaki, Wakolbinger, \& Kettinger, 2013), e poucas são as empresas que possuem sistemas para esse fim (Hazen, Overstreet, Hall, Huscroft, Hanna, 2015).

Em países em desenvolvimento, a implementação da LR enfrenta ainda maiores desafios. Ausência de incentivos econômicos, legislação ou baixa capacidade de enforcement por parte do setor público (Li, Jayaraman, Paulraj, Shang, 2016), conflitos na cadeia reversa e a falta de conscientização da população (Demajorovic, Augusto, Souza, 2016; Zurbrügg, Caniato, \& Vaccari, 2014) pouco estimulam o investimento das empresas nesses países.

No Brasil, a Lei n. 12.305/2010, que instituiu a Política Nacional de Resíduos Sólidos (PNRS), fomentou a LR e um conjunto de ações e procedimentos para a coleta e destinação final de resíduos, incluindo os eletroeletrônicos. Para tanto, estabeleceu regulamentações, acordos setoriais e termos de compromisso para atingimento das metas (Ministério do Meio Ambiente, 2018). A resistência dos representantes em assinar o acordo setorial favoreceu o baixo interesse dos membros integrantes da cadeia de eletroeletrônicos em investir e implementar programas em larga escala de sistemas de LR para os resíduos eletroeletrônicos (Demajorovic, Augusto, Souza, 2016): Apesar do baixo comprometimento e interesse do setor de eletroeletrônico em promover a LR, no final de 2019, quase 10 anos depois de promulgada a PNRS, o acordo setorial foi assinado.

Nesse processo, pouca ou nenhuma atenção foi dada a como a TI poderia contribuir para alcançar as metas propostas no acordo setorial. Assim, este trabalho tem como objetivo analisar a contribuição de sistemas de informação e as tecnologias de rastreamento para a LR de cadeias de resíduos de eletroeletrônicos no cenário brasileiro.

\section{REFERENCIAL TEÓRICO}

Nesta seção, é discutida a importância da TI e das tecnologias de rastreamento para a implementação de programas de LR. Esse embasamento teórico proporcionou o desenvolvimento de um modelo de análise, os instrumentos de coleta de dados e fundamentou a análise e discussão dos resultados.

\section{Os sistemas de informação e as tecnologias de rastreamento na cadeia de suprimentos}

O gerenciamento da cadeia de suprimentos verde, Green Supply Chain Management (GSCM), inclui o design de produto (Gmelin \& Seuring, 2014), a análise do ciclo de vida, as operações verdes que abarcam a LR e a gestão de resíduos sólidos. Os produtos são projetados levando em consideração a conservação dos recursos, o aproveitamento dos resíduos na remanufatura, no reúso e na reciclagem (Liu, Zhu, \& Seuring, 2017).

Três aspectos são considerados barreiras para a implementação da GSCM: altos custos; esforços de coordenação e complexidade; insuficiência ou falha de comunicação na cadeia de suprimentos (Seuring \& Müller, 2008). Os sistemas de informação verde - Green Information Systems (GIS) fornecem as informações necessárias para decisões sobre ecodesign, em termos de material e consumo de energia, reutilização, reciclagem e destinação pós-consumo (Li et al., 2016). Esses sistemas provêm a interface em ambiente compartilhado para apoiar uma gestão sustentável (Yang, Sun, Zhang, Wang, 2018). 
Os fluxos de bens e informação no GSCM devem ser interligados e coordenados de modo a envolver vários departamentos da organização, assim como fornecedores e clientes, para melhorar o desempenho de toda a cadeia (Morgan, Richey, \& Autry, 2016). Atualmente, os fluxos de informação no GSCM estão se expandindo, e a combinação das competências em LR com TI possibilita um melhor desempenho do fluxo reverso de produtos (Morgan et al., 2016). Os processos de reciclagem tornam-se mais eficientes e há a destinação correta dos resíduos (Prajogo, Olhager, 2012; Morgan, Richey, Tokman, Deffe, 2018).

A LR possui várias atividades, iniciando pela devolução do produto usado, que pode ser direcionada aos revendedores ou fabricantes, por meio de programas de retorno, ou a pontos de coleta públicos ou privados. Após a coleta, é feita a inspeção para classificação nas diversas categorias: reparo, reúso, remanufatura ou reciclagem (Agrawal, Singh, \& Murtaza, 2015; Dhanda \& Hill, 2005). Nessa área, existem vários estudos para o uso de TI para melhorar a eficiência no gerenciamento de resíduos (Król, Nowakowski, \& Mrówczyńska, 2016). Os gestores podem melhorar a comunicação dos parceiros, integrar informações sobre as atividades da empresa e melhorar a capacidade de resposta (Daugherty, Richey, Genchev, Chen, 2005).
Estudos mostram que a perda de informação é um dos principais obstáculos para recuperar o valor dos produtos retornados (Madaan, Kumar, \& Chan, 2012). A TI prove informações precisas sobre o status, a localização e a condição dos produtos em movimento na cadeia de suprimentos, para devolver os produtos nos locais e tempos corretos (Jayaraman, Ross, \& Agarwal, 2008). Portanto, sistemas compatíveis que facilitam a troca de informações entre organizações ou entre unidades devem ser empregados (Huscroft, Hazen, Hall, Hanna, 2013a).

No Gerenciamento de Recuperação de Produtos (Product Recovery Management - PRM)-(Toyasaki et al., 2013), podem existir os Sistemas de Recuperação de Produto com Informações Intensivas (Information-intensive Product Recovery System - IPRS), que fornecem informações sobre os produtos para possibilitar a recuperação, a automatização de processos manuais e redução de tempo e de custos de classificação. Esses sistemas necessitam de informações mais precisas sobre identificação, uso e condição do produto para possibilitar um processo de LR mais eficiente (Toyasaki et al., 2013). A Figura 1 mostra como o PRMS estabelece a integração entre bases de dados e todas as atividades da cadeia de LR.

\section{Figura 1. Sistema de gerenciamento de recuperação de produtos}

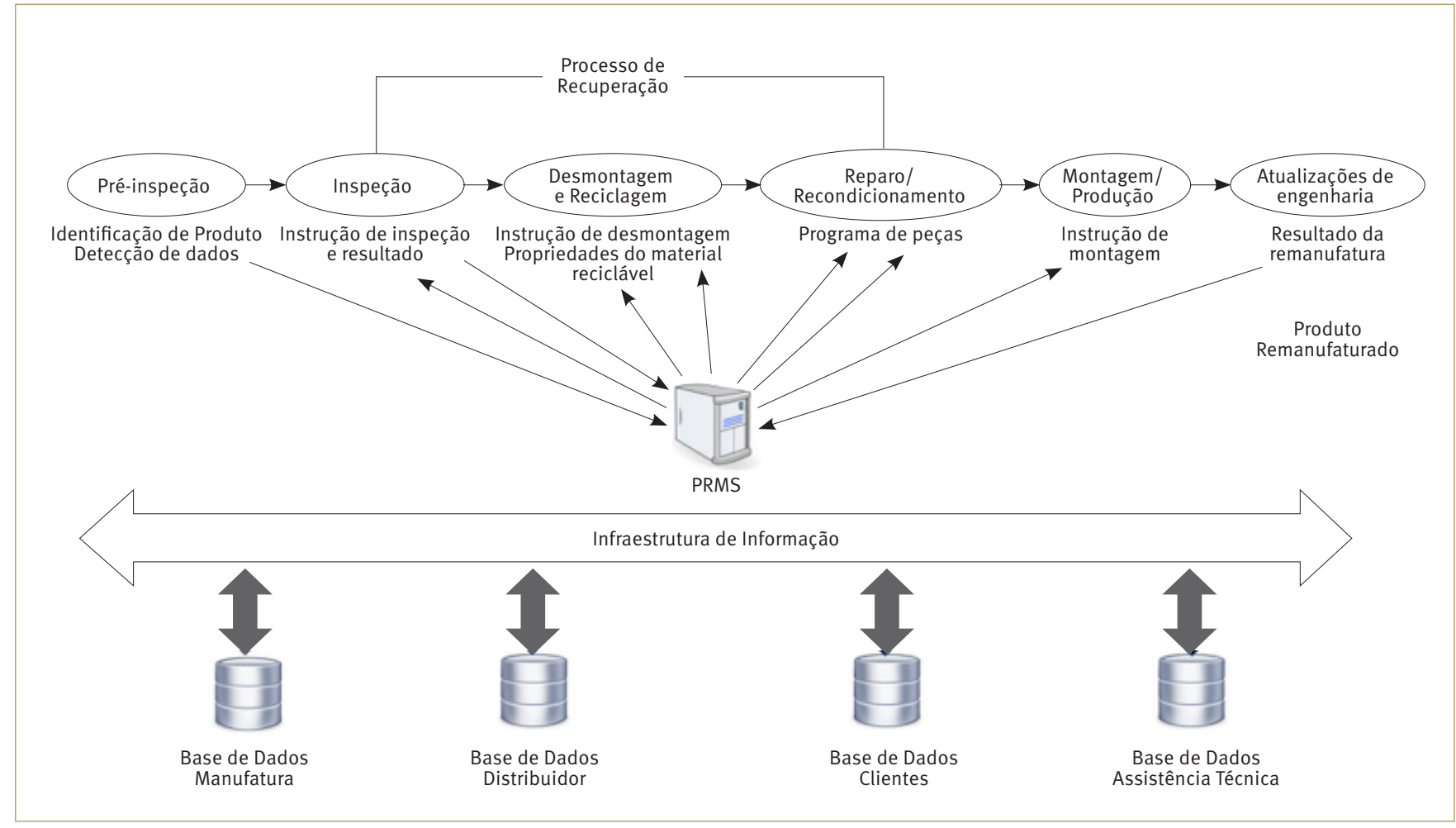


O PRMS precisa ser desenvolvido para permitir o acesso aos bancos de dados por todos os stakeholders, possibilitando a integração de sistemas por meio de informações padronizadas e uso de tecnologias de rastreamento como RFID, código de barras ou chip integrado. No caso da desmontagem, o PRMS pode contribuir com informações sobre os materiais que compõem o produto. As informações sobre remanufatura devem apresentar instruções de reparo e remontagem (Um et al., 2008) e gerar relatórios para a tomada de decisões que levem à melhoria do desempenho ambiental da cadeia e cumprimento de metas (Green, Zelbst, Meacham, Bhadauria, 2012a).

Essas tecnologias sempre foram usadas nos processos de automação da logística direta, e agora fornecem também informação para o fluxo reverso, reduzindo o tempo de classificação de produtos (Toyasaki et al., 2013). Uma das vantagens do RFID é o fato de que muitos itens podem ser simultaneamente identificados de maneira remota, sem a necessidade de direcionamento para cada um deles (Kumar, 2014; Parlikad \& Mcfarlane, 2007). Mesmo tendo as etiquetas RFID um custo maior, tem crescido a utilização dessa tecnologia nas linhas de montagem e nos centros de distribuição (Um et al., 2015). 0 código de barras é uma tecnologia óptica apenas para leitura de dados, o que significa que, uma vez impressos, os dados não podem ser alterados, e o leitor de código de barras precisa estar próximo e direcionado para o produto ou embalagem. É uma tecnologia muito utilizada em logística direta, de baixo custo, e é universal (Musa et al., 2014). Os chips integrados são a tecnologia mais avançada, presente em vários produtos eletroeletrônicos e uma das mais caras. As informações capturadas podem realimentar as áreas de pesquisa e desenvolvimento para prover produtos mais ecoeficientes (Um et al., 2015).

A decisão mais correta para que o produto seja reparado, reutilizado, recondicionado ou reciclado depende de vários parâmetros, entre eles: a qualidade do produto; a data de fabricação e de venda; e as condições a que o produto foi submetido em todo o seu ciclo de vida, como temperatura ambiente, umidade, taxa de utilização e manutenção (Parlikad \& Mcfarlane, 2007). 0 uso de tecnologia RFID, para fazer o acompanhamento, começa definindo em qual estágio do ciclo de vida se encontra o produto, se está em Beginning of Life (BOL) - Começo da vida do produto, Middle of Life (MOL) - Meia-vida do ciclo, ou End of Life (EOL) - Fim de vida do produto. Essa definição sobre o ciclo de vida do produto possibilita selecionar sua destinação entre as alternativas de reúso, remanufatura ou reciclagem. Se um produto está no BOL, provavelmente pode ser encaminhado ao reúso, bastando que seja consertado. Se está no MOL, a destinação provável é a remanufatura, e, em EOL, seria destinado a reciclagem (Um, Stroud, Suh, 2015).
As estratégias de compartilhamento de informações via RFID permitem um controle de estoque e inventário mais preciso, com monitoramento em tempo real (Shin, Suh, Stroud, Yoon, 2017), que ajuda a coordenar as políticas de estoque entre fornecedores de material reciclado e fabricantes na cadeia de suprimentos, reduzindo custos ambientais e econômicos e adaptando rapidamente às mudanças no sistema (Green, Zelbst, Sower, Reyes, 2012b; Nativi \& Lee, 2012).

\section{MODELO DE ANÁLISE}

Esta pesquisa parte do pressuposto de que os sistemas de recuperação de produtos intensivos em informação podem colaborar com a LR, aumentando a eficiência e diminuindo custos (Daugherty et al., 2005; Jayaraman et al., 2008; Olorunniwo \& Li, 2010; Toyasaki et al., 2013). O Sistema para Gerenciamento de Recuperação de Produtos - Product Recovery Management System (PRMS) passa a integrar os sistemas e as bases de dados de todos os envolvidos no Gerenciamento da Cadeia de Suprimentos Verde - GSCM, como fabricante, distribuidores, revendas, assistências técnicas e centros de coleta e reciclagem, assim como o uso de sensores para identificação e obtenção de informação dos produtos como RFID, ou código de barras e chips integrados. A Figura 2 apresenta o fluxo direto e reverso de produtos, com captura de informações em todas as áreas principais, com o uso tecnologias de rastreamento e coleta de informações (Nativi \& Lee, 2012; Nowakowski, 2018). A proposta deste modelo de análise é apresentar uma síntese da aplicação de tecnologias de rastreamento e sistemas de informação no fluxo reverso, como discutido no referencial teórico.

$\mathrm{Na}$ logística direta, esses sensores são utilizados no processo de fabricação com a introdução das tecnologias de rastreamento nos produtos (Nowakowski, 2018; Um et al., 2015). No processo de distribuição, os produtos são rastreados e os sistemas, atualizados com informações sobre as entregas. Nas revendas, a informação coletada provê aos sistemas os níveis de vendas, que retroalimentam os estoques dos distribuidores e dos fabricantes que podem planejar a produção (Um et al., 2015).

Os sistemas de informação com coletores de informação de produtos também podem contribuir para o desenvolvimento e planejamento da rede de LR (Green et al., 2012a; Liu et al., 2017; Müller \& Seuring, 2007). Nos centros de coleta, inspeção e destinação, o uso de sistemas de informação pode identificar materiais perigosos, quantidade e peso dos vários componentes, além de fornecer instruções para desmontagem e informações sobre a destinação correta (Nowakowski, 2018). Esses 
sistemas podem também beneficiar processos manuais pouco automatizados (Nativi \& Lee, 2012; Parlikad \& Mcfarlane, 2007; Toyasaki et al., 2013). As informações capturadas nos centros de coleta atualizam as bases de dados e fornecem dados para o desenvolvimento de metas para o fluxo reverso (Li et al., 2016; Olorunniwo \& Li, 2010). O uso da TI para o rastreamento e captura de informações, como o RFID (Nativi \& Lee, 2012; Nowakowski, 2018), permite avaliar a destinação mais adequada de acordo com as características do produto e seu tempo de vida. Seu direcionamento pode ser para a fábrica, no caso de remanufatura, ou para a assistência técnica para reparo, ou para um distribuidor

\section{Figura 2. Modelo conceitual de análise}

ou uma revenda para reúso (Nativi \& Lee, 2012; Nowakowski, 2018; Srivastava, 2008; Um et al., 2015).

Os dados coletados também retroalimentam a base de dados de pesquisa e desenvolvimento de novos produtos, trazendo informações importantes para melhoria de produtos, embalagens e ecodesign (Agrawal et al., 2015; Gmelin \& Seuring, 2014; Green et al., 2012a; Li et al., 2016). Para isso, as bases devem ser integradas e o acesso, compartilhado entre os principais participantes da cadeia para a tomada de decisão e a criação de relatórios gerenciais (Green et al., 2012a; Um et al., 2015).

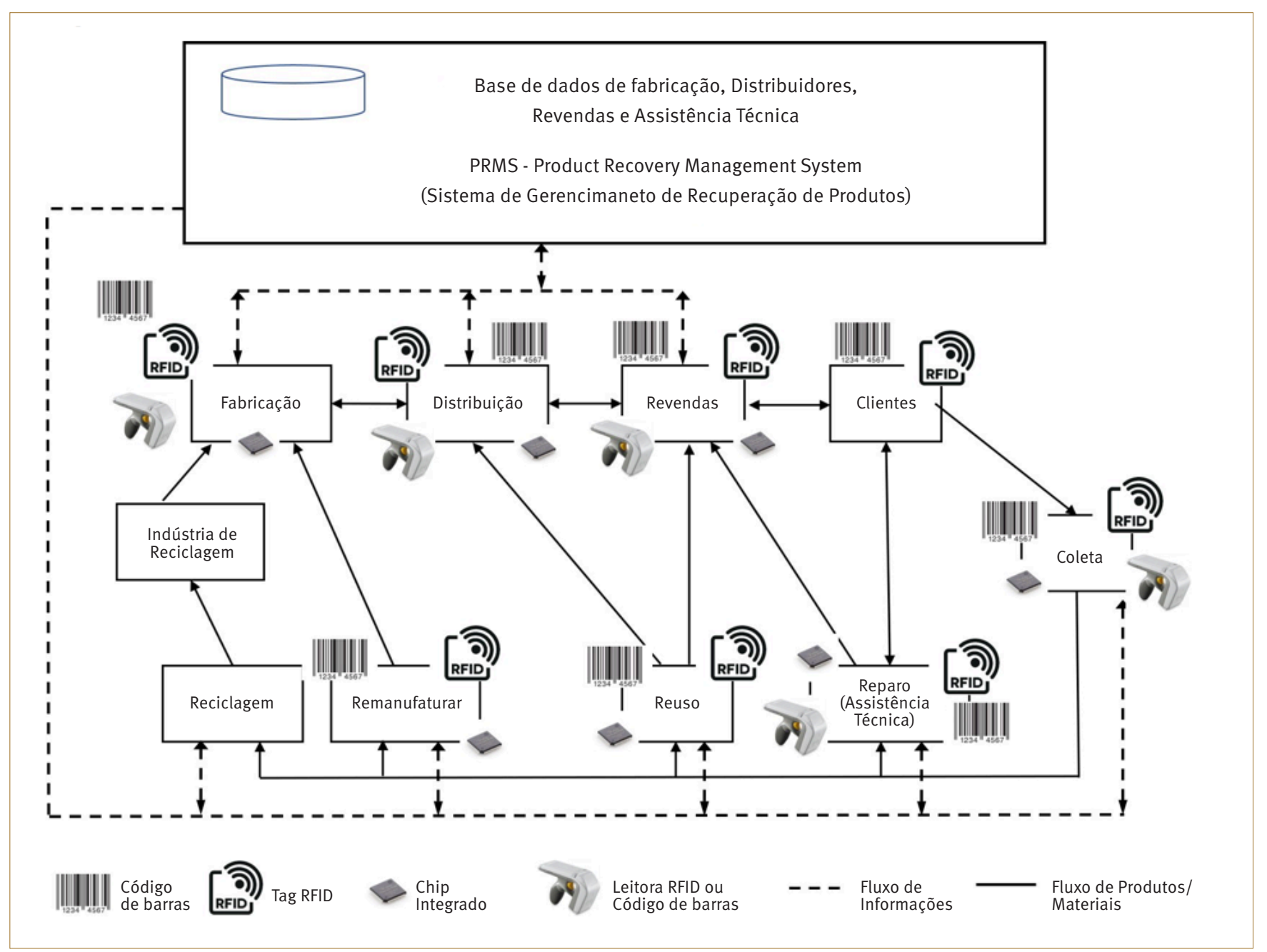

\section{MÉTODO DE PESQUISA}

Os estudos de caso podem ser utilizados para diferentes tipos de pesquisa, como exploração, construção de teoria, teste de teoria e extensão e refinamento de teoria (Voss, Tsikriktsis, \& Frohlich, 2002). A abordagem metodológica foi o estudo de caso único integrado com várias unidades de análise incorporadas (Yin, 2017). A amostra teórica é composta de unidades de análise sobre as 
quais os dados do estudo são coletados (Yin, 2017). A validade do estudo requer a seleção de pelo menos quatro casos com base nos quais serão definidos padrões (Eisenhardt, 1989) que devem ser escolhidos com o objetivo de predizer resultados semelhantes ou contrastantes (Yin, 2017). As organizações pesquisadas foram escolhidas de modo a representar os principais atores envolvidos na cadeia reversa de REEE (Resíduos de Equipamentos
Eletroeletrônicos) no cenário brasileiro nos segmentos de computadores pessoais, notebooks, tablets e impressoras. Assim, as unidades de análise foram dois fabricantes, um distribuidor, um varejista, uma empresa gestora de LR e um centro de inovação de LR, uma assistência técnica, uma empresa de reciclagem e uma cooperativa. Características das empresas e dos entrevistados são apresentadas no Quadro 1.

\section{Quadro 1. Características das empresas pesquisadas}

\begin{tabular}{|c|c|}
\hline Empresa Pesquisada & Características \\
\hline Fabricante B & $\begin{array}{l}\text { Empresa sediada nos EUA com aproximadamente } 55 \text { mil colaborares mundialmente. No Brasil, tem cerca de } \\
500 \text { funcionários com sede em São Paulo e atua no mercado corporativo e doméstico em todo o território } \\
\text { nacional. Possui vários distribuidores e revendas. }\end{array}$ \\
\hline Gestora de Logística Reversa & $\begin{array}{l}\text { Empresa brasileira sediada em São Paulo com cerca de } 30 \text { funcionários e cobertura nacional. Atende os } \\
\text { principais fabricantes de REE. }\end{array}$ \\
\hline $\begin{array}{l}\text { Centro de Inovação e } \\
\text { Reciclagem }\end{array}$ & $\begin{array}{l}\text { Empresa brasileira, com participação de multinacionais da área de tecnologia, sediada em São Paulo, com } \\
\text { cerca de } 90 \text { funcionários e cobertura nacional. Atende os principais fabricantes de REE. }\end{array}$ \\
\hline Distribuidor & $\begin{array}{l}\text { Distribuidor de produtos na área de tecnologia com sede nos EUA e controlado por grupo chinês, com } 33 \text { mil } \\
\text { funcionários mundialmente. No Brasil, está sediado em São Paulo com aproximadamente } 600 \text { funcionários e } \\
\text { atuação nacional. Seus clientes são fabricantes na área de tecnologia e eletrônicos. }\end{array}$ \\
\hline Assistência Técnica & $\begin{array}{l}\text { Assistência técnica de equipamentos de informática com sede em São Paulo e atuação na grande São Paulo } \\
\text { com cerca de } 60 \text { funcionários, é multimarcas. }\end{array}$ \\
\hline Cooperativa & $\begin{array}{l}\text { Cooperativa de reciclagem de eletroeletrônicos, com atuação na Grande São Paulo, com } 30 \text { funcionários, e é } \\
\text { multimarcas. }\end{array}$ \\
\hline Recicladora & $\begin{array}{l}\text { Recicladora de eletroeletrônicos e metais com atuação na grande São Paulo e com } 60 \text { funcionários. Atende o } \\
\text { mercado cooperativo e os principais fabricantes de REE. }\end{array}$ \\
\hline
\end{tabular}

Em todas as unidades analisadas, a pesquisa realizada focou as tecnologias envolvidas na captura e rastreamento de produtos para o fluxo reverso e os sistemas de informação existentes para o processamento dessas informações. 0 Fabricante A está presente apenas no mercado corporativo, enquanto o Fabricante B atua nos mercados corporativo e doméstico. As fontes de evidência utilizadas na coleta de dados foram os documentos, as entrevistas semiestruturadas e a observação direta. A utilização de múltiplas fontes de evidências, que garantem validade do construto da pesquisa, e as diversas fontes de informação, quando convergentes, contribui para a confiabilidade dos dados (Yin, 2017).

Para os estudos de caso, os documentos são importantes, pois corroboram e aumentam a evidência de outras fontes (Yin, 2017). Os documentos utilizados neste estudo de caso foram os relatórios de sustentabilidade, sites das empresas e documentos fornecidos pelos entrevistados, enquanto a observação direta é frequentemente útil para proporcionar informação adicional sobre o tópico sendo estudado e se aplica durante a pesquisa de campo (Yin, 2017). A observação direta foi realizada na Cooperativa, na Recicladora, no Varejista e no Centro de Inovação e Reciclagem. As questões das entrevistas semiestruturadas foram fundamentadas nas categorias teóricas levantadas na revisão da literatura, a saber: o fluxo de produtos na logística direta e reversa; rede de LR; processamento, armazenamento e captura de informação de produtos; integração de sistemas entre departamentos e integrantes da cadeia de suprimentos; uso de tecnologia de captura de informação de produtos, como RFID, código de barras ou chips integrados, conforme mostrado no Quadro 2. 


\section{Quadro 2. Questões da entrevista relacionadas às categorias teóricas da pesquisa}

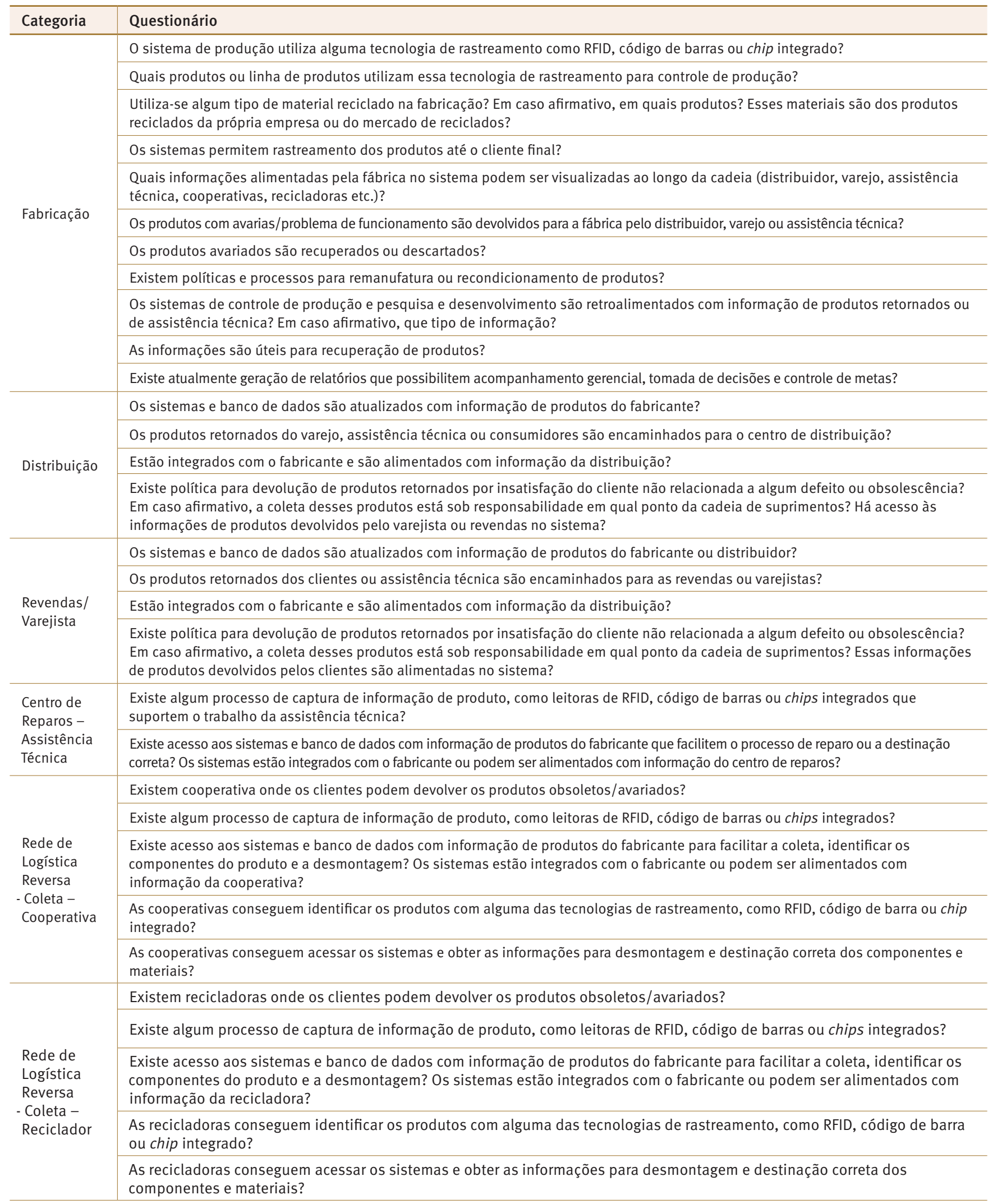


O público-alvo das entrevistas e dos questionários foram os gestores responsáveis pela área de sustentabilidade das empresas, LR e operações. As entrevistas foram gravadas e transcritas. Os documentos utilizados neste estudo de caso foram os relatórios de sustentabilidade e sites das empresas. Além disso, durante as entrevistas, alguns gestores completaram as informações verbais com registro em arquivos da empresa pesquisada. A observação direta foi realizada na Cooperativa, na Recicladora, no Varejista e no Centro de Inovação e Reciclagem, com aplicação do protocolo de observação desenvolvido especialmente para este estudo. 0 Quadro 3 apresenta a codificação das entrevistas, da análise documental e da observação direta.

Quadro 3. Codificação das entrevistas, documentos e observação

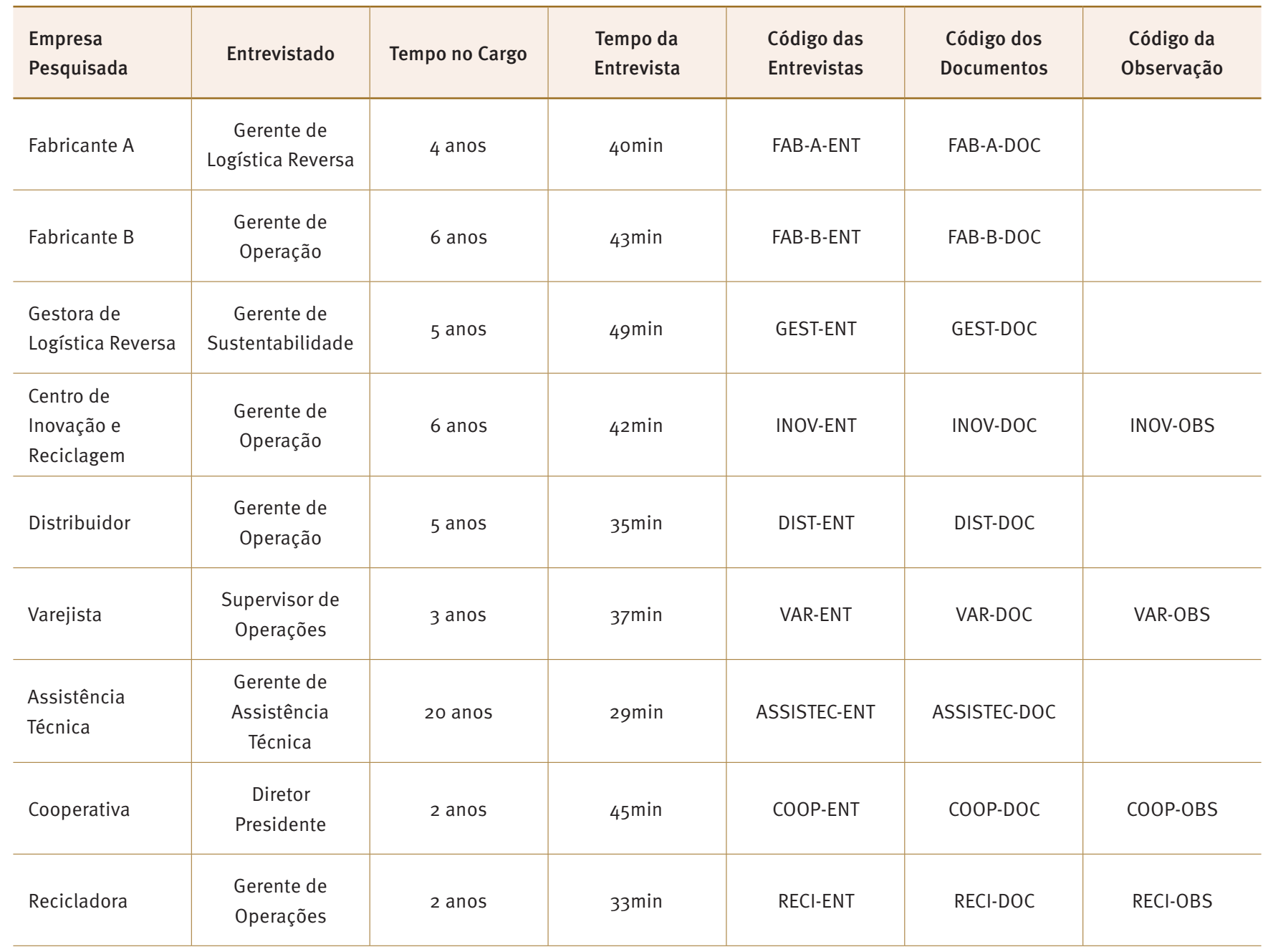

A análise e o tratamento dos dados foram realizados por meio da a triangulação de dados de três fontes - entrevistas, da análise documental e observação - que permitiu o encadeamento das evidências e contribuiu para a validade do construto (Yin, 2017). Como o processo de obtenção dos dados gera uma grande quantidade de informações, a análise possibilita aos pesquisadores lidar com esse volume de informações (Eisenhardt, 1989), e, para este estudo, foi usada a estratégia de seguir as categorias teóricas estabelecidas na pesquisa.

\section{ANÁLISE E DISCUSSÃO DOS RESULTADOS}

A pesquisa, no entanto, indicou um distanciamento entre o modelo de análise, que sintetizou as informações sobre sistemas de informação e das tecnologias de rastreamento obtidas na revisão da literatura, e os dados empíricos. Os principais resultados das entrevistas, da análise documental e da observação direta foram codificados e apresentados no Quadro 4. 


\section{Quadro 4. Resumo dos resultados da pesquisa nas unidades de análise}

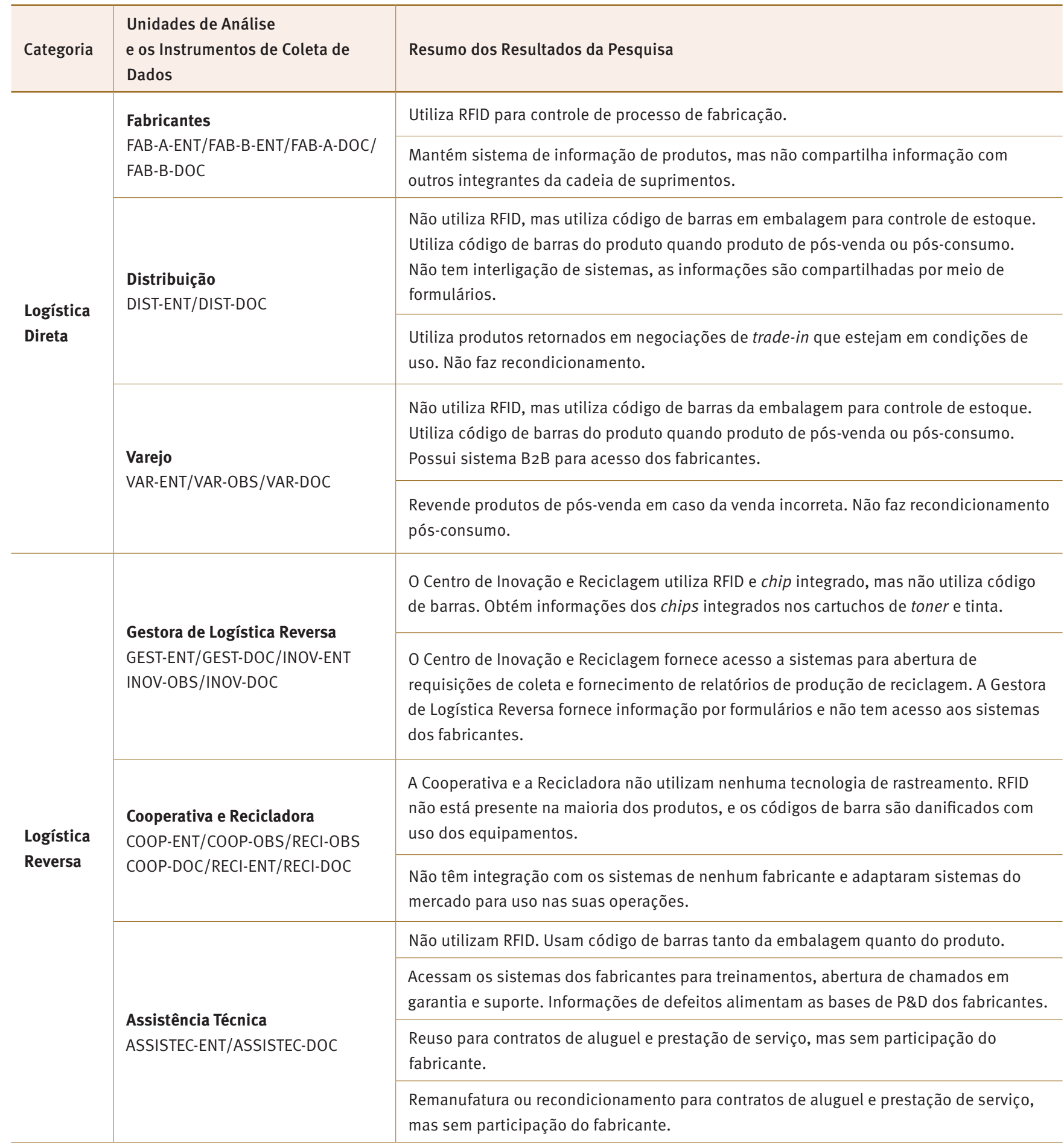

A pesquisa mostrou que os sistemas de informação estão presentes em todas as áreas da cadeia de suprimentos direta e reversa, mas ainda distantes de soluções interligadas que poderiam permitir compartilhamento de informações mais eficiente. 0 fluxo de informação deveria fornecer o necessário para tornar os processos de reciclagem mais eficientes e possibilitar a destinação correta dos resíduos, como aponta a literatura (Morgan et al., 2018; Olorunniwo \& Li, 2010). A Figura 3 ilustra a síntese dos resultados obtidos na pesquisa de campo. 
Figura 3. Síntese dos resultados obtidos na pesquisa de campo

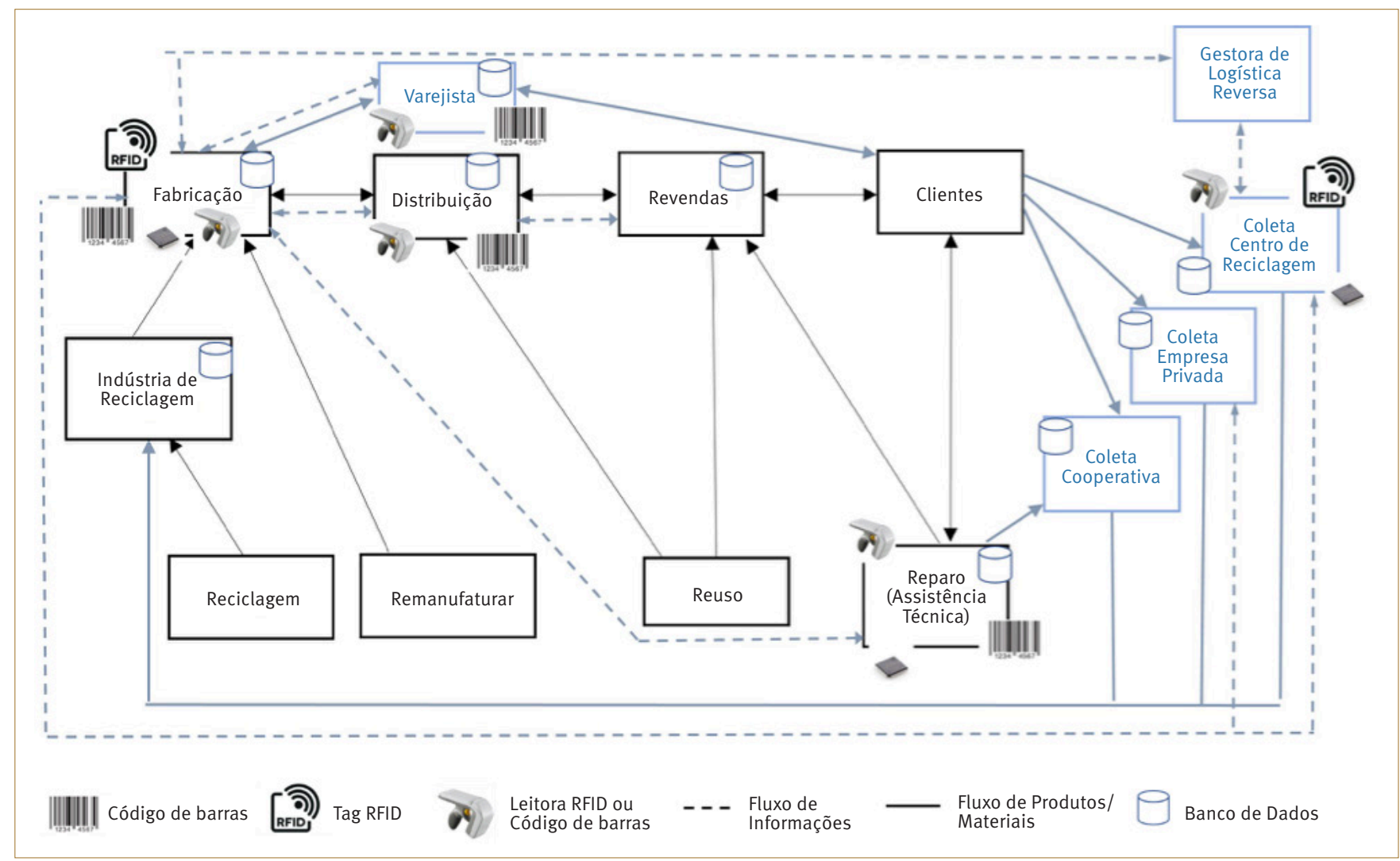

Nota: os varejistas compram direto dos fabricantes e vendem para consumidores. As revendas compram de distribuidores e vendem em todos os mercados

Na logística direta, o distribuidor e os fabricantes utilizam controles, que são relatórios desenvolvidos pelos distribuidores. Os sensores são utilizados no processo de fabricação com a introdução das tecnologias de rastreamento, como o RFID, códigos de barras ou chips incorporados, corroborando diversos aspectos levantados na revisão da literatura (Nowakowski, 2018; Um et al., 2015). Os fabricantes desenvolveram um sistema para acesso das assistências técnicas para agilizar o conserto de equipamentos em garantia. A Assistência Técnica acessa o sistema dos fabricantes quando há a necessidade de compra de peças ou suporte para produtos em garantia. Esses sistemas integrados podem melhorar a capacidade de resposta dos parceiros, como mencionado em outros estudos (Daugherty et al., 2005).

A necessidade do Varejista de manter seus estoques abastecidos e na quantidade correta, para evitar aumento de custos em armazenagem, levou à criação de um sistema B2B para acesso on-line e em tempo real pelos fabricantes. Essa solução proporcionou rapidez na movimentação do estoque e abastecimento de produtos. Entretanto, ainda é considerada pouco eficiente pelos usuários, considerando a velocidade das transações comerciais atuais. Estudos mostram que sistemas com informações coletadas por meio de tecnologias de rastreamento contribuiriam de modo mais eficaz para localização, entregas e vendas (Nativi \& Lee, 2012; Shin et al., 2017), proporcionando informações atualizadas para melhor controle de estoque (Green et al., 2012b) e planejamento da produção (Um et al., 2015)

A pesquisa indicou que o desenvolvimento de sistemas e a interligação entre eles ocorrem quando há impacto nas vendas. Cada membro desenvolve sua própria estrutura interligada com outros elos da cadeia para atender a propósitos específicos. As bases de dados não estão ainda interligadas, perdendo informação significativas para recuperação de valor como apresentado na literatura (Madaan et al., 2012). Pesquisas anteriores mostram que os sistemas necessitam de investimentos em tecnologia (Ajamieh et al., 2016; Huscroft, Hazen, Hall, Skipper, Hanna, 2013b), para que as empresas desenvolvam competências relacionadas a integração e disseminação das informações (Huscroft et al., 2013a; Toyasaki et al., 2013), para apoiar a gestão ambiental (Yang et al., 2018).

Quanto à LR, não existem sistemas prontos no mercado. Todos tiveram que adaptar ou criar seu próprio sistema. A pesquisa revelou um estágio muito incipiente de adoção dessas 
tecnologias no mercado nacional. O processo de controle de peças e componentes não é contemplado pelas soluções de software de logísticas disponíveis, dificultando o fluxo reverso e a remanufatura, o que corrobora estudos anteriores (Morgan et al., 2016). Mesmo nessas condições, o Centro de Inovação e Reciclagem oferece acesso on-line para os clientes, que podem requisitar coletas e ter acesso à quantidade em peso de produtos processados. A Gestora de LR repassa aos clientes as informações de produção também em forma de relatórios, mas está desenvolvendo um sistema que permita acesso on-line e em tempo real. A Cooperativa e a Recicladora também adaptaram seus sistemas para melhorar a tomada de decisões relacionada ao fluxo reverso de produtos, como sugere a literatura (Green et al., 2012a; Liu et al., 2017), mas sem interligação com a cadeia, o que não contribui para a eficiência do processo como um todo.

No Brasil, os sistemas como PRM e IPRS, discutidos no referencial teórico (Toyasaki et al., 2013), que fornecem informações para a identificação e tempo de vida útil, ainda estão distantes de serem utilizados. Esses sistemas tornariam mais eficientes os programas de reúso, recondicionamento ou remanufatura, segundo alguns autores (Agrawal et al., 2015; Dhanda \& Hill, 2005). Nas assistências técnicas, o que define o reparo ou o recondicionamento é a disponibilidade e o custo das peças. O Distribuidor compra e recondiciona produtos de clientes corporativos, mas sem a participação dos fabricantes. Como apontado pela literatura, a Tl é um enorme obstáculo para as empresas, por falta de recursos e conhecimento adequado na área (Bouzon, Miguel, \& Rodriguez, 2014), o que provoca falha de comunicação na cadeia e processos ineficientes (Seuring \& Müller, 2008).

A Cooperativa realiza algum recondicionamento de produtos em pequeno volume para revenda ou uso próprio. As informações de reparos que podem ajudar no desenvolvimento de produtos ficam a cargo das assistências técnicas. Os Distribuidores e Varejistas repassam informações de defeitos somente quando o produto é devolvido no prazo de sete dias, conforme o Código de Defesa do Consumidor brasileiro. Não existe retroalimentação por parte dos recicladores para desenvolver produtos com ecodesign, que facilitem a desmontagem e a não utilização de materiais que contaminam outros. Essa retroalimentação de informação, diferente da literatura (Gmelin \& Seuring, 2014; Li et al., 2016), não acontece entre os fabricantes de eletroeletrônicos e recicladores, aumentando os custos da remanufatura e reciclagem. Componentes de produtos que utilizam solda ou parafusos, por exemplo, aumentam o tempo da desmontagem e, consequentemente, a mão de obra empregada no processo.
A pesquisa mostrou que o uso tecnologia de rastreamento, apresentado em estudos sobre o tema (Um \& Suh, 2015), como o RFID para LR, existe em apenas uma linha de produtos do Fabricante B, e o Centro de Inovação e Reciclagem faz a leitura quando o produto chega ao Centro de Reciclagem. O Fabricante A e Fabricante B utilizam na produção, mas nenhum outro participante da cadeia de suprimentos utiliza essa tecnologia para controle de estoque. A questão do custo ainda é apontada como a principal barreira, reforçando os argumentos de Hazen et al. (2015). Outros atores da cadeia admitem que, no futuro, essa tecnologia possa ser utilizada em produtos com maior valor agregado, principalmente por facilitar a leitura a distância (Kumar, 2014). Estudos mostram que o uso de tecnologia de RFID permitiria um melhor controle sobre o inventário com monitoramento em tempo real e o compartilhamento de informações (Green, Zelbst, Sower, Bellah, 2016; Nativi \& Lee, 2012) para obter maiores benefícios ambientais e econômicos (Jayaraman et al., 2008).

O código de barras é amplamente utilizado na logística direta, principalmente em embalagens. O código de barras do produto é mais utilizado em assistências técnicas e também em situações de pós-venda ou pós-consumo para varejistas e distribuidores. Na LR, o código de barras não é utilizado. Como não existe nenhum tipo de interligação com sistemas dos fabricantes, o código de barras não identifica o produto, e, consequentemente, não existe informação, como modelo, fabricante, peso, tipos de materiais e informação de desmontagem, que poderia ser útil para a reciclagem, como sugere a literatura (Musa et al., 2014). Além disso, muitas vezes, o código de barras do produto está danificado.

Para o gestor da Cooperativa, se os fabricantes utilizassem um código de barras ou RFID que fosse universal, a identificação dos produtos para reciclagem seria facilitada e ajudaria muito na operação, aumentando a produtividade com ganho de escala. Com uma identificação rápida, a empresa poderia obter informações de desmontagem, identificação dos materiais e componentes perigosos, diminuindo custos e simplificando o processo, como sugerem alguns estudos (Nativi \& Lee, 2012; Nowakowski, 2018; Parlikad \& Mcfarlane, 2007).

O gestor do Centro de Inovação e Reciclagem destacou que o RFID poderia ser usado para o planejamento do fornecimento de reciclados e melhor controle de estoque. 0 fabricante, tendo conhecimento prévio da quantidade de material reciclado disponível, ajusta seu estoque com o fornecedor de insumos e, consequentemente, diminui o consumo de matéria-prima virgem, como concluíram algumas pesquisas (Nativi \& Lee, 2012).

O uso do chip integrado foi observado em cartuchos de toner e tintas. A Assistência Técnica que fornece esse suprimento 
para clientes com contrato de serviço ou o Centro de Inovação e Reciclagem que tem contrato com o Fabricante A e Fabricante $B$ controlam e auditam esses suprimentos lendo a informação contida no chip para passar aos fabricantes. Os fabricantes têm interesse especial na reciclagem desses suprimentos para evitar que sejam recondicionados e retornem ao mercado paralelo. $A$ pesquisa não identificou o uso de chip integrado em mais nenhum eletroeletrônico, pelo menos com a intenção de rastreabilidade voltada para a LR, como apontado na literatura (Um et al., 2008).

Para alguns atores da cadeia, a assinatura do acordo setorial será o elemento catalisador para a expansão significativa dos investimentos em sistemas e tecnologias de rastreamento na estrutura implementada para o cumprimento das metas, que corrobora estudos sobre o tema (Dou, Zhu, \& Sarkis, 2014; Green et al., 2012a).

\section{CONCLUSÕES}

O objetivo desta pesquisa foi analisar a contribuição de sistemas de informação e das tecnologias de rastreamento para a LR de cadeias de resíduos de eletroeletrônicos no contexto brasileiro. Os resultados da pesquisa mostram que os sistemas de informação estão presentes em todos os pontos da cadeia de suprimentos direta e reversa, mas a interligação de sistemas não é efetiva na cadeia, e a implementação dessas tecnologias ainda é incipiente no mercado brasileiro. Alguns atores da cadeia percebem o potencial das tecnologias de rastreamento, com codificação universal, para identificação de produtos e acesso a sistemas que informem o modelo, o tempo estimado de vida útil, características como peso, tipos de materiais e componentes perigosos. Também veem nessas tecnologias uma alternativa para reduzir custos no processo, tornando mais eficientes a desmontagem e a reciclagem, assim como uma oportunidade a ser usada para planejamento de produção e controle de estoque de materiais reciclados.

Por outro lado, as principais barreiras identificadas na pesquisa estão relacionadas ao fato de tecnologias de rastreamento utilizadas no fluxo reverso serem percebidas de maneira bastante distinta do seu uso na logística direta. A partir do estudo de caso realizado, prevalece uma visão de que o emprego de tais tecnologias no fluxo reverso não contribui para o aumento das vendas e a lucratividade de todas as empresas da cadeia, mas eleva custos operacionais e faltam recursos para investir. Além disso, muitas empresas tiveram que desenvolver seu próprio sistema, em razão de não existir no mercado um sistema que integre toda a cadeia. Por fim, a falta de conhecimento de gestores dessas tecnologias de rastreamento para o fluxo reverso chamou atenção em alguns pontos da cadeia. Essas barreiras dificultam o fluxo de informações e tornam os processos ineficientes, retardando os investimentos e as parcerias estratégicas das empresas do setor em países em desenvolvimento como o Brasil.

Nesse aspecto, a identificação das principais barreiras na utilização de tecnologias de rastreamento no fluxo reverso é uma contribuição gerencial significativa desta pesquisa. A recente assinatura do acordo setorial de eletroeletrônicos, em outubro de 2019, poderá ser um elemento importante para potencializar os benefícios das tecnologias de rastreamento e na superação das barreiras encontradas nesta pesquisa para efetivação em larga escala da LR de REEE no cenário brasileiro.

A contribuição teórica desta pesquisa é o modelo analítico, que sintetiza os principais elementos que constituem as vantagens do uso dessas tecnologias e sistemas. A revisão da literatura mostrou a contribuição, em vários aspectos, dos sistemas de informação e das tecnologias de rastreamento para a implementação de programas de LR, que já são utilizados em alguns países, com potencial para aumentar a eficiência e superar as barreiras de países em desenvolvimento.

Dessa forma, o desenvolvimento de novas pesquisas sobre avaliação de investimentos e de custos de sistemas de informação e tecnologias de rastreamento em programas de LR surge como um tema emergente e importante para futuros estudos.

\section{REFERÊNCIAS}

Agrawal, S., Singh, R. K., \& Murtaza, Q. (2015, April). A literature review and perspectives in reverse logistics. Resources, Conservation and Recycling, 97, 76-92. doi: 10.1016/j.resconrec.2015.02.009

Ajamieh, A., Benitez, J., Braojos, J., Gelhard, C. . (2016, October). IT infrastructure and competitive aggressiveness in explaining and predicting performance. Journal of Business Research, 69(10), 46674674. doi: 10.1016/j.jbusres.2016.03.056

Bouzon, M., Miguel, P., \& Rodriguez, C. (2014, August). Managing end of life products: A review of the literature on reverse logistics in Brazil. Management of Environmental Quality: An International Journal, 25(5), 564-584. doi: 10.1108/MEQ-04-2013-0027

Daugherty, P. J.; Richey, R. G.; Genchev, S. E.; Chen, H.; (2005, March). Reverse logistics: Superior performance through focused resource commitments to information technology. Transportation Research Part E: Logistics and Transportation Review, 41(2), 77-92. doi: 10.1016/j.tre.2004.04.002

Demajorovic, J., Augusto, E. E. F., \& Souza, M. T. S. (2016, April). Reverse logistics of e-waste in developing countries: Challenges and prospects for the Brazilian model. Ambiente \& Sociedade, 19(2), 117136. doi:10.1590/1809-4422asoc141545V1922016 
Dhanda, K. K., \& Hill, R. P. (2005, January). The role of information technology and systems in reverse logistics: A case study. International Journal of Technology Management, 31(1-2), 140-151. doi: $10.1504 /$ IJTM.2005.006628

Dou, Y., Zhu, Q., \& Sarkis, J. (2014, March). Evaluating green supplier development programs with a grey-analytical network process-based methodology. European Journal of Operational Research, 233(2), 420-431. doi: 10.1016/j.ejor.2013.03.004

Eisenhardt, K. M. (1989, October). Building theories from case study research. The Academy of Management Review, 14(4), 3-36. doi: $10.2307 / 258557$

Gmelin, H., \& Seuring, S. (2014, April). Determinants of a sustainable new product development. Journal of Cleaner Production, 69(3), 1-9. doi: 10.1016/j.jclepro.2014.01.053

Green, K. W.; Zelbst, P. J.; Meacham, J.; Bhadauria, V. S.; (2012a, April). Green supply chain management practices: Impact on performance. Supply Chain Management: An International Journal, 17(3), 290-305. doi: $10.1108 / 13598541211227126$

Green, K. W.; Zelbst, P. J.; Sower, V. E.; Reyes, P. M.; (2012b, February). Impact of RFID on manufacturing effectiveness and efficiency. International Journal of Operations \& Production Management, 32(3), 329-350. doi: 10.1108/01443571211212600

Green, K. W.; Zelbst, P. J.; Sower, V. E.; Bellah J. C.; (2016, September). Impact of radio frequency identification technology on environmental sustainability. Journal of Computer Information Systems, 57(3), 269277. doi: $10.1080 / 08874417.2016 .1184029$

Hazen, B. T.; Overstreet, R. E.; Hall, D. J.; Huscroft, J. R.; Hanna, J. B.; (2015, April). Antecedents to and outcomes of reverse logistics metrics. Industrial Marketing Management, 46(1), 160-170. doi: 10.1016/j.indmarman.2015.01.017

Hazen, B. T., \& Byrd, T. A. (2012, January). Toward creating competitive advantage with logistics information technology. International Journal of Physical Distribution \& Logistics Management, 42(1), 8-35. doi: $10.1108 / 09600031211202454$

Huscroft, J. R.; Hazen, B. T.; Hall, D. J.; Hanna, J. B.; (2013a, August). Task technology fit for reverse logistics performance. The International Journal of Logistics Management, 24(2), 230-246. doi: 10.1108/ijlm02-2012-0011

Huscroft, J. R.; Hazen, B. T.; Hall, D. J.; Skipper, J. B.; Hanna, J. B.; (2013b, November). Reverse logistics: Past research, current management issues, and future directions. The International Journal of Logistics Management, 24(3), 304-327. doi:: 10.1108/IJLM-04-2012-0024

Jayaraman, V., Ross, A., \& Agarwal, A. (2008, January). Role of information technology and collaboration in reverse logistics supply chains. International Journal of Logistics Research and Applications, 11(6), 409-425. doi: 10.1080/13675560701694499

Król, A., Nowakowski, P., \& Mrówczyńska, B. (2016, April). How to improve WEEE managementNovel approach in mobile collection with application of artificial intelligence. Waste Management, 50(1), 222 233. doi: 10.1016/j.wasman.2016.02.033

Kumar, S. (2014, September). A knowledge based reliability engineering approach to manage product safety and recalls. Expert Systems with Applications, 41(11), 5323-5339. doi: 10.1016/j.eswa.2014.03.007
Li, S.; Jayaraman, V.; Paulraj, A.; Shang, K.; (2016, January). Proactive environmental strategies and performance: Role of green supply chain processes and green product design in the Chinese high-tech industry. International Journal of Production Research, 54(7), 2136 2151. doi: $10.1080 / 00207543.2015 .1111532$

Liu, Y., Zhu, Q., \& Seuring, S. (2017, March). Linking capabilities to green operations strategies: The moderating role of corporate environmental proactivity. International Journal of Production Economics, 187(1), 182-195. doi: 10.1016/j.ijpe.2017.03.007

Madaan, J., Kumar, P., \& Chan, F. T. S. (2012, June). Decision and information interoperability for improving performance of product recovery systems. Decision Support Systems, 53(3), 448-457. doi 10.1016/j.dss.2012.02.011

Ministério do Meio Ambiente. (2018). Sistema Nacional de Informações sobre a Gestão de Resíduos Sólidos. Recuperado de http://www. sinir.gov.br/web/guest/plano-nacional-de-residuos-solidos

Morgan, T. R.; Richey, R. G.; Tokman, M.; Defee, C.; (2018, February). Resource commitment and sustainability: A reverse logistics performance process model. International Journal of Physical Distribution \& Logistics Management, 48(2), 164-182. doi: 10.1108/ IJPDLM-02-2017-0068

Morgan, T. R., Richey, R. G., Jr., \& Autry, C. W. (2016, April). Developing a reverse logistics competency. International Journal of Physical Distribution \& Logistics Management, 46(3), 293-315. doi: 10.1108/ IJPDLM-05-2014-0124

Müller, M., \& Seuring, S. (2007, April). Reducing information technologybased transaction costs in supply chains. Industrial Management \& Data Systems, 107(4), 484-500. doi: 10.1108/02635570710740652

Musa, A., Gunasekaran, A., \& Yusuf, Y. (2014, January). Supply chain product visibility: Methods, systems and impacts. Expert Systems with Applications, 41(1), 176-194. doi: 10.1016/j.eswa.2013.07.020

Nativi, J. J., \& Lee, S. (2012, April). Impact of RFID information-sharing strategies on a decentralized supply chain with reverse logistics operations. International Journal of Production Economics, 136(2), 366-377. doi: 10.1016/j.ijpe.2011.12.024

Nowakowski, P. (2018, January). A novel, cost efficient identification method for disassembly planning of waste electrical and electronic equipment. Journal of Cleaner Production, 172(1), 2695-2707. doi: 10.1016/j.jclepro.2017.11.142

Olorunniwo, F. O., \& Li, X. (2010, September). Information sharing and collaboration practices in reverse logistics. Supply Chain Management: An International Journal, 15(6), 454-462. doi: $10.1108 / 13598541011080437$

Parlikad, A. K., \& Mcfarlane, D. (2007, November). RFID-based product information in end-of-life decision making. Control Engineering Practice, 15(11), 1348-1363. doi: 1016/j.conengprac.2006.08.008

Prajogo, D.; Olhager, J. Supply chain integration and performance: The effects of long-term relationships, information technology and sharing, and logistics integration. International Journal of Production Economics, Melbourne, v. 135, n. 1, p. 514-522, jan. 2012. doi: 10.1016/j.ijpe.2011.09.001

Seuring, S., \& Müller, M. (2008, October). From a literature review to a conceptual framework for sustainable supply chain management. Journal of Cleaner Production, 16(15), 1699-1710. doi: 10.1016/j. jclepro.2008.04.020 
Shin, S.-J.; Suh, S. H.; Stroud, I.; Yoon, S.; (2017, August). Processoriented life cycle assessment framework for environmentally conscious manufacturing. Journal of Intelligent Manufacturing, 28(6), 1481-1499. doi: 10.1007/s10845-015-1062-4

Srivastava, S. K. (2008, February). Value recovery network design for product returns. International Journal of Physical Distribution \& Logistics Management, 38(4), 311-331. doi: 10.1108/09600030810875409

Toyasaki, F., Wakolbinger, T., \& Kettinger, W. J. (2013, February). The value of information systems for product recovery management. International Journal of Production Research, 51(4), 1214-1235. doi: 10.1080/00207543.2012.695090

Um, J., Stroud, I. A., \& Suh, S. H. (2015, September). Development and evaluation of customisation process for ubiquitous product recovery management system. International Journal of Computer Integrated Manufacturing, 28(9), 903-919. doi: 10.1080/0951192X.2014.941404

Um, J., \& Suh, S.-H. (2015, April). Design method for developing a product recovery management system based on life cycle information. International Journal of Precision Engineering and Manufacturing-
Green Technology, 2(2), 173-187. doi: 10.1007/\$40684-015-0022-y

Um, J., Yoon, J. S., \& Suh, S. H. (2008, August). An architecture design with data model for product recovery management systems. Resources, Conservation and Recycling, 52(10), 1175-1184. doi: 10.1016/j. resconrec.2008.06.001

Voss, C., Tsikriktsis, N., \& Frohlich, M. (2002). Case research in operations management. International Journal of Operations \& Production Management, 22(2), 195-219. doi: 10.1108/01443570210414329

Yang, Z.; Sun, J.; Zhang, Y.; Wang, Y.; (2018, June). Peas and carrots just because they are green? Operational fit between green supply chain management and green information system. Information Systems Frontiers, 20(3), 627-645. doi: 10.1007/s10796-016-9698-y

Yin, R. K. (2017). Case study research and applications: Design and methods (6th ed.). Sage PublicationCalifórnia, EUA.

Zurbrügg, C., Caniato, M., \& Vaccari, M. (2014, February). How assessment methods can support solid waste management in developing countries: A critical review. Sustainability, 6(2), 545-570. doi: $10.3390 /$ su6020545

\section{CONTRIBUIÇÃO DOS AUTORES}

O Odair Oliveira Bernardo e a Maria Tereza Saraiva de Souza participaram da conceitualização e abordagem teórica-metodológica. O Odair Oliveira Bernardo realizou a revisão teórica e a coleta de dados. Todos os autores trabalharam na análise de dados, redação e revisão final do artigo. 